ing of the main constituents is thus of great importance. As an example Fig. 5 shows the PA monitoring of ethanol $\left(\mathrm{C}_{2} \mathrm{H}_{5} \mathrm{OH}\right)$-vapour present in the exhaust stream of a pharmaceutical production plant besides numerous other volatile organic compounds. The PA measurements were performed periodically at selected $\mathrm{CO}_{2}$ laser transitions yielding ethanol and methanol concentrations simultaneously. In general, good agreement between the photoacoustically derived concentrations and independent gaschromatographic (GC) measurements (represented by $\mathrm{x}$ in Fig. 5 ) is obtained under steady state conditions. The PA data are not systematically higher than the GC data as could be supposed from the results in Fig. 5 , but the time resolution offered by PAS is better and can be exploited for the monitoring of even short-term concentration fluctuations.

\section{Perspectives}

Since photoacoustics involves optical, thermal and acoustical phenomena, photoacoustic and photothermal sensing schemes are ideally suited for studying various material properties. Apart from non-spectroscopic applications like e.g. thermal wave imaging which today represents an important technique for the non-destructive evaluation (NDE) of materials, laser-PA spectroscopy offers great potential for applications ranging from surface physics and chemistry to environmental sciences. The few examples discussed indicate the wide gamut of PAS applications that can be expected.

\section{Acknowledgement}

The author is grateful to S. Bernegger and P.L. Meyer for their collaboration. Our photoacoustic studies were funded by the Swiss National Science Foundation (National Research Program 14) and ETH Zurich.

\section{FURTHER READING}

Zharov V.P. and Letokhov V.S., Laser Optoacoustic Spectroscopy, Springer Ser. Opt. Sciences, Vol. 37 (Springer Berlin, Heidelberg) 1986.

Tam A.C., Rev. Mod. Phys. 58 (1986) 381. 431.

Sigrist M.W., J. Appl. Phys. 60 (1986) R83R121.

Rothberg L., J. Phys. Chem. 91 (1987) 3467-3474.

Photoacoustic and Photothermal Phenomena, Eds. P. Hess and J. Pelzl, Springer Ser. Opt. Sciences, Vol. 58 (Springer Berlin, Heidelberg) 1988.

Sigrist M.W., Bernegger S. and Meyer P.L., Infrared Phys. 29 (1989) 805-814.

Photoacoustic, Photothermal and Photochemical Processes in Gases, Ed. P. Hess, Top. Curr. Phys., Vol. 46 (Springer Berlin, Heidelberg) 1989.

Photoacoustic, Photothermal and Photochemical Processes at Surfaces and in Thin Films, Ed. P. Hess, Top. Curr. Phys. Vol. 47 (Springer Berlin, Heidelberg) 1989.

\title{
EQEC: 2nd European Quantum Electronics Conference
}

\section{Dresden, 28 August - 1 September 1989}

Following EQEC-1, the first Divisional Conference of the Quantum Electronics Division held in Hannover in 1988, EQEC-2 took place in Dresden's Town Hall under the auspices of the GDR Academy of Sciences, the Friedrich Schiller University, Jena, and the Physical Society of the GDR.

The four plenary papers highlighted present mainstreams in quantum electronics. Akhmanov (Moscow) reported on non-linear optics in 2-dimensional systems, discussing non-linear wave dynamics, large scale transverse wave interaction, optical turbulence and spatial squeezing. These abstract concepts were beautifully illustrated by experimental results shown in an impressive movie. Welling (Hannover) reviewed the many routes towards very-short-wavelength lasers and their potential applications. Glauber (Cambridge) discussed the theory of radiating atoms in resonant cavities, while Paul (Berlin) addressed a basic issue in quantum optics, namely interference between independent single photons, touching upon basic issues in quantum measurement theory.

An important contribution in the area of laser physics was made by Bimberg (W. Berlin) who reported on gain switching of semiconductor lasers where picosecond pulses can be produced by driving the laser with a much longer electrical pulse in such a way that only the first relaxation oscillation spike occurs. Dunn (St. Andrews) discussed the use of excimer lasers to pump optical parametric oscillators (OPO's) in the visible. Generally speaking OPO's have been of marginal importance during the past 25 years but they are now rapidly gaining popularity owing to new nonlinear materials and new pump sources becoming available. Prior (Rehovot) addressed current models used to describe laser fluctuations, namely phase diffusion, random phase jumps and telegraph phase noise, generalizing these into a non-Markovian stochastic jump model. Distributed feedback gas lasers were analysed in their own right, and as a model system for DFB solid state lasers, by Kneubühl (Zurich). DFB gas lasers allow easy tests of theory since their parameters are much better controlled than in the case of a DFB semiconductor laser. Lasers were also addressed by Ovaevski (Moscow) in the context of the intriguing analogy between lasing action and superconductivity that can be made by comparing Cooper pairs with photons.

Regarding non-linear optical materials, Kobayashi (Tokyo) considered the problems which arise when one has to assess the many new materials (particularly polymers) which are presently being developed for fast optical switches and optical logic. He introduced a simple method based on measuring the transmission spectrum of a polymer layer sandwiched between two transparent electrodes. Stockman (Novosibirsk) gave a very interesting presentation of the giant optical non-linearities of fractal clusters such as aggregates of colloidal gold.

Walther (Max-Planck-Institut, Garching) reported on recent single-atom experiments which allow many early "Gedanken" experiments to be performed in the laboratory. Such experiments may resolve some basic issues in quantum physics since ensemble averaging is no longer present. Another significant contribution in a related area included the report by Chebotaev (Novosibirsk) on synchronisation in quantum transitions, making connections with Ramsey fringes, photon echoes and free induction decay. Possible applications would be super high resolution spectroscopy and high-speed atomic memory systems. In a theoretical paper Stenholm (Helsinki) touched upon the issue of Landau-Zener crossing during a laser assisted collision. For reasons not yet understood the range of validity of the "classical" Landau-Zener result is apparently much larger than expected. Finally, Noordam (Amsterdam) reported recent results obtained in producing electronic wave packets in Rydberg atoms using picosecond laser excitation. A full set of abstracts of all invited and contributed papers has been published in Volume 13D of the Europhysics Conference Abstracts series which is available from the EPS Secretariat.

J.P. Woerdman, Leiden 